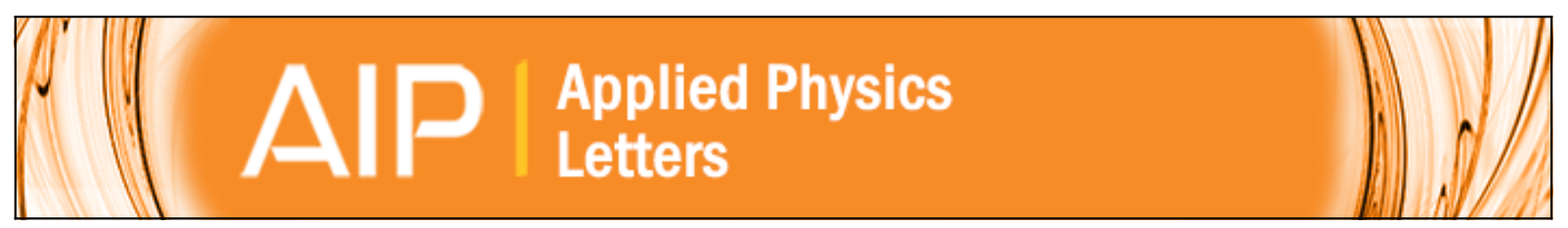

\title{
Perpendicular magnetic anisotropy in Ta|Co40Fe40B20|MgAl2O4 structures and perpendicular CoFeB|MgAl2O4|CoFeB magnetic tunnel junction
}

B. S. Tao, D. L. Li, Z. H. Yuan, H. F. Liu, S. S. Ali, J. F. Feng, H. X. Wei, X. F. Han, Y. Liu, Y. G. Zhao, Q. Zhang, Z. B. Guo, and X. X. Zhang

Citation: Applied Physics Letters 105, 102407 (2014); doi: 10.1063/1.4895671

View online: http://dx.doi.org/10.1063/1.4895671

View Table of Contents: http://scitation.aip.org/content/aip/journal/apl/105/10?ver=pdfcov

Published by the AIP Publishing

\section{Articles you may be interested in}

Reducing spin-torque switching current by incorporating an ultra-thin Ta layer with CoFeB free layer in magnetic tunnel junctions

Appl. Phys. Lett. 105, 242411 (2014); 10.1063/1.4903825

Perpendicular magnetic anisotropy in $\mathrm{Ta} / \mathrm{Co} 2 \mathrm{FeAl} / \mathrm{MgO}$ multilayers

J. Appl. Phys. 114, 063905 (2013); 10.1063/1.4818326

Effect of Mg interlayer on perpendicular magnetic anisotropy of CoFeB films in $\mathrm{MgO} / \mathrm{Mg} / \mathrm{CoFeB} / \mathrm{Ta}$ structure Appl. Phys. Lett. 101, 122414 (2012); 10.1063/1.4754118

Effect of thickness of $\mathrm{MgO}$, Co-Fe-B, and Ta layers on perpendicular magnetic anisotropy of [Ta/Co60Fe20B20/MgO]5 multilayered films

J. Appl. Phys. 111, 07C111 (2012); 10.1063/1.3673408

The perpendicular anisotropy of Co40Fe40B20 sandwiched between $\mathrm{Ta}$ and $\mathrm{MgO}$ layers and its application in CoFeB/MgO/CoFeB tunnel junction

Appl. Phys. Lett. 99, 012502 (2011); 10.1063/1.3605564

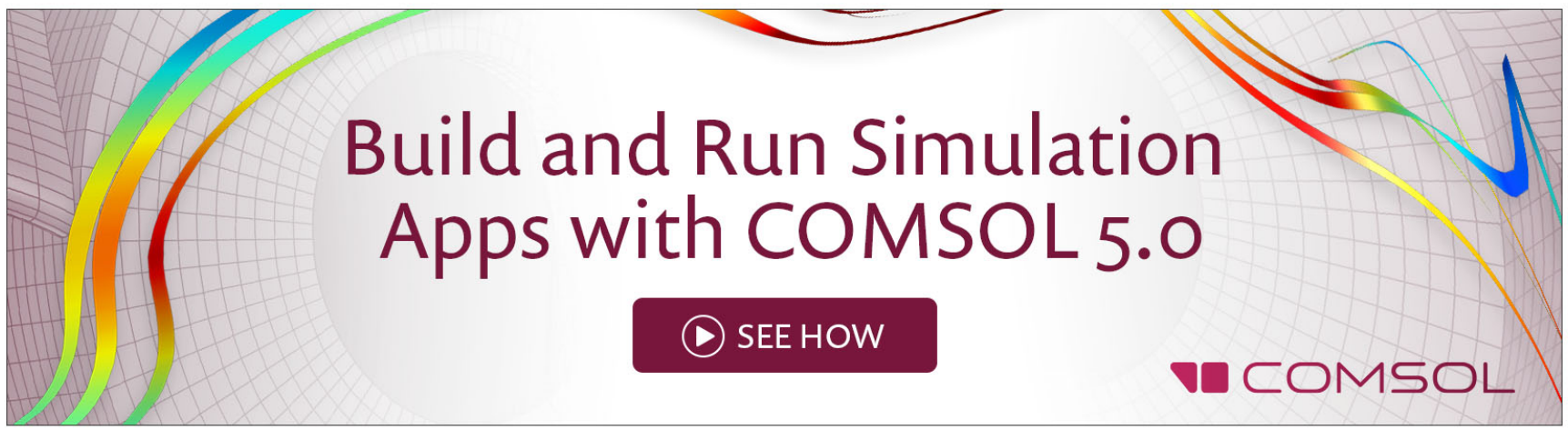




\title{
Perpendicular magnetic anisotropy in $\mathrm{Ta}\left|\mathrm{Co}_{40} \mathrm{Fe}_{40} \mathrm{~B}_{20}\right| \mathrm{MgAl}_{2} \mathrm{O}_{4}$ structures and perpendicular $\mathrm{CoFeB}\left|\mathrm{MgAl}_{2} \mathrm{O}_{4}\right| \mathrm{CoFeB}$ magnetic tunnel junction
}

\author{
B. S. Tao, ${ }^{1}$ D. L. Li, ${ }^{1}$ Z. H. Yuan, ${ }^{1}$ H. F. Liu, ${ }^{1}$ S. S. Ali, ${ }^{1}$ J. F. Feng,,${ }^{1}$ H. X. Wei, ${ }^{1}$ X. F. Han, ${ }^{1, a)}$ \\ Y. Liu, ${ }^{2}$ Y. G. Zhao, ${ }^{2}$ Q. Zhang, ${ }^{3}$ Z. B. Guo, ${ }^{3}$ and X. X. Zhang ${ }^{3,4}$ \\ ${ }^{1}$ Beijing National Laboratory of Condensed Matter Physics, Institute of Physics, Chinese Academy of Sciences, \\ Beijing 100190, China \\ ${ }^{2}$ Department of Physics and State Key Laboratory of Low-Dimensional Quantum Physics, \\ Tsinghua University, Beijing 100084, China \\ ${ }^{3}$ Advanced Nanofabrication, Imaging and Characterization Core Laboratory, King Abdullah University \\ of Science and Technology (KAUST), Thuwal 239955, Kingdom of Saudi Arabia \\ ${ }^{4}$ Division of Physical Science and Engineering, King Abdullah University of Science and Technology, \\ Thuwal 239955, Kingdom of Saudi Arabia
}

(Received 9 June 2014; accepted 22 August 2014; published online 11 September 2014)

\begin{abstract}
Magnetic properties of $\mathrm{Co}_{40} \mathrm{Fe}_{40} \mathrm{~B}_{20}(\mathrm{CoFeB})$ thin films sandwiched between Ta and $\mathrm{MgAl}_{2} \mathrm{O}_{4}$ layers have been systematically studied. For as-grown state, $\mathrm{Ta} / \mathrm{CoFeB} / \mathrm{MgAl}_{2} \mathrm{O}_{4}$ structures exhibit good perpendicular magnetic anisotropy (PMA) with interface anisotropy $K_{\mathrm{i}}=1.22 \mathrm{erg} / \mathrm{cm}^{2}$, which further increases to $1.30 \mathrm{erg} / \mathrm{cm}^{2}$ after annealing, while $\mathrm{MgAl}_{2} \mathrm{O}_{4} / \mathrm{CoFeB} / \mathrm{Ta}$ multilayer shows in-plane magnetic anisotropy and must be annealed in order to achieve PMA. For bottom CoFeB layer, the thickness window for PMA is from 0.6 to $1.0 \mathrm{~nm}$, while that for top CoFeB layer is between 0.8 and $1.4 \mathrm{~nm}$. Perpendicular magnetic tunnel junctions (p-MTJs) with a core structure of $\mathrm{CoFeB} / \mathrm{MgAl}_{2} \mathrm{O}_{4} /$ $\mathrm{CoFeB}$ have also been fabricated and tunneling magnetoresistance ratio of about $36 \%$ at room temperature and $63 \%$ at low temperature have been obtained. The intrinsic excitations in the p-MTJs have been identified by inelastic electron-tunneling spectroscopy. (C) 2014 AIP Publishing LLC.

[http://dx.doi.org/10.1063/1.4895671]
\end{abstract}

Perpendicular magnetic tunnel junctions (p-MTJs) with ferromagnetic electrodes possessing perpendicular magnetic anisotropy (PMA) have attracted great interests due to their advantages for spintronic applications, especially for highdensity spin-transfer torque magnetic random access memory (STT-MRAM). ${ }^{1-4}$ High thermal stability in the order of nanometers, low critical switching current density and high tunneling magnetoresistance (TMR) ratio are required for a memory cell in STT-MRAM. ${ }^{2,4}$ A variety of materials with perpendicular easy axis have been explored to satisfy the requirements mentioned above, ${ }^{2,4}$ among which ultra thin CoFeB film with a PMA has attracted a great deal of attention since it fulfils all the requirements at the same time. ${ }^{5-10}$ $\mathrm{MgO}$ based MTJs with an in-plane anisotropy have been extensively studied and a high TMR ratio have been obtained. ${ }^{11-13}$ However, the lattice mismatch between $\mathrm{MgO}$ and typical ferromagnetic electrodes leads to a rapid reduction of TMR with bias voltage and a low breakdown voltage, ${ }^{14,15}$ which is unfavorable for practical applications. Recently, spinel oxide $\mathrm{MgAl}_{2} \mathrm{O}_{4}$ has been explored as tunnel barrier to further improve the performance of MTJs with inplane anisotropy due to its small lattice mismatch with typical ferromagnetic electrodes. ${ }^{14-19}$ High TMR ratio and weak bias voltage dependence of TMR have been reported in epitaxial $\mathrm{MgAl}_{2} \mathrm{O}_{4}$ barrier based MTJs grown on single crystal $\mathrm{MgO}$ substrate, where $\mathrm{MgAl}_{2} \mathrm{O}_{4}$ barrier was formed by plasma oxidation of $\mathrm{Mg} / \mathrm{Al}$ bilayer $^{14}$ or $\mathrm{Mg}$-Al alloy. ${ }^{18,19}$ So far, there are no reports about p-MTJs with $\mathrm{MgAl}_{2} \mathrm{O}_{4}$ barrier.

\footnotetext{
${ }^{\text {a) }}$ Author to whom correspondence should be addressed. Electronic mail: xfhan@iphy.ac.cn
}

For $\mathrm{Ta} / \mathrm{CoFeB} / \mathrm{MgO}$ structure, the interfacial PMA is attributed to hybridization of transition metal $3 d$ and $\mathrm{O} 2 p$ orbitals. $^{20,21}$ Moreover, the Ta/CoFeB interface also plays a key role in the PMA of thin $\mathrm{CoFeB}$ films, ${ }^{6}$ which can be enhanced by adjusting the underlayers. ${ }^{8,9}$ However, it should be noted that the under- or over-oxidized interface can destroy the PMA, ${ }^{21,22}$ making it difficult to obtain PMA in $\mathrm{CoFeB} / \mathrm{MgAl}_{2} \mathrm{O}_{4}$ system with $\mathrm{MgAl}_{2} \mathrm{O}_{4}$ formed by oxidation of $\mathrm{Mg} / \mathrm{Al}$ bilayer or $\mathrm{Mg}-\mathrm{Al}$ alloy.

In this work, magnetic properties of $\mathrm{CoFeB}$ thin films sandwiched between $\mathrm{Ta}$ and $\mathrm{MgAl}_{2} \mathrm{O}_{4}$ layers have been systematically investigated, where $\mathrm{MgAl}_{2} \mathrm{O}_{4}$ film is formed by RF sputtering from a sintered stoichiometric $\mathrm{MgAl}_{2} \mathrm{O}_{4}$ target to avoid the oxidation problem. Ta is adopted as under and cap layers because of its critical contribution to PMA. Our experimental results show that good PMA can be realized in thin $\mathrm{CoFeB}$ film by optimizing the thickness of $\mathrm{CoFeB}$ layer and post-annealing treatment. Based on the PMA optimization of $\mathrm{CoFeB}$ thin films at either side of $\mathrm{MgAl}_{2} \mathrm{O}_{4}$ layer, $\mathrm{p}$ MTJs with a core structure of $\mathrm{CoFeB} / \mathrm{MgAl}_{2} \mathrm{O}_{4} / \mathrm{CoFeB}$ have been fabricated and studied.

Film stacks were deposited using an ULVAC magnetron sputtering system with a base pressure of $1.0 \times 10^{-6} \mathrm{~Pa}$. Two series of multilayers: Ta (5)/CoFeB $(t=0.6-1.6) / \mathrm{MgAl}_{2} \mathrm{O}_{4}$ (2)/Ta (5) and $\mathrm{Ta}(5) / \mathrm{MgAl}_{2} \mathrm{O}_{4}(2) / \mathrm{CoFeB}(t=0.8-1.8) / \mathrm{Ta}$ (5), corresponding to bottom and top electrodes in MTJs, and MTJ stack with the structure of Ta (5)/Ru (30)/Ta (5)/CoFeB (1.0)/ $\mathrm{MgAl}_{2} \mathrm{O}_{4}(2) / \mathrm{CoFeB}(1.2) / \mathrm{Ta}(5) / \mathrm{Ru}$ (5) (numbers are nominal thickness in nanometer) were deposited on thermally oxidized Si wafers. All the samples were annealed at $300{ }^{\circ} \mathrm{C}$ in vacuum with an out-of-plane magnetic field of 
8000 Oe for half an hour. Magnetic properties were characterized by vibrating sample magnetometer (VSM) and superconducting quantum interference device (SQUID). The MTJ was patterned into junctions with size of $10 \times 20 \mu \mathrm{m}^{2}$ using ultraviolet photolithography combined with $\mathrm{Ar}$ ion beam etching and transport properties were measured by fourprobe method. Rutherford backscattering spectrometry (RBS) was carried out to characterize element distribution in the MTJ.

Fig. 1 shows the magnetic hysteresis loops of Ta (5)/ $\mathrm{CoFeB}(t) / \mathrm{MgAl}_{2} \mathrm{O}_{4}(2) / \mathrm{Ta}(5 \mathrm{~nm})$ (bottom electrode) with $t$ varying from 0.6 to $1.2 \mathrm{~nm}$. The red line with solid circles and blue line with solid squares represent perpendicular and inplane hysteresis curves, respectively. For the as-grown state, the samples with $t=0.6$ and $1.0 \mathrm{~nm}$ exhibit an out-of-plane easy axis, showing a well-squared shape with a squareness $(\mathrm{SQ})=1$ and 0.8 , respectively, while the sample with $t=1.2 \mathrm{~nm}$ shows an in-plane easy axis. After annealing at $300^{\circ} \mathrm{C}$, the in-plane saturation field decreases from 7000 to 2000 Oe for sample with $t=0.6 \mathrm{~nm}$ but increases from 2000 to $2500 \mathrm{Oe}$ for sample with $t=1.0 \mathrm{~nm}$. Also, the SQ decreases from 1 to 0.4 for the sample with $t=0.6 \mathrm{~nm}$ and increases from 0.8 to 1.0 for the sample with $t=1.0 \mathrm{~nm}$. For the sample with $t=1.2 \mathrm{~nm}$, the easy axis still lies in plane, but the perpendicular saturation field decreases from 3000 to 2000 Oe. The above results show that PMA after annealing is degraded in the sample with $t=0.6 \mathrm{~nm}$ but enhanced slightly in the samples with $t=1.0$ and $1.2 \mathrm{~nm}$.

Fig. 2 shows the magnetic hysteresis loops of Ta (5)/ $\mathrm{MgAl}_{2} \mathrm{O}_{4}(2) / \mathrm{CoFeB}(t) / \mathrm{Ta}$ (5) (top electrode) with $t$ varying from 1.0 to $1.6 \mathrm{~nm}$. For the as-grown state, all the samples exhibit an in-plane easy axis but the perpendicular saturation field decreases with decreasing $t$, as shown in Figs. 2(a)-2(c), which indicates that magnetization has an increasing perpendicular
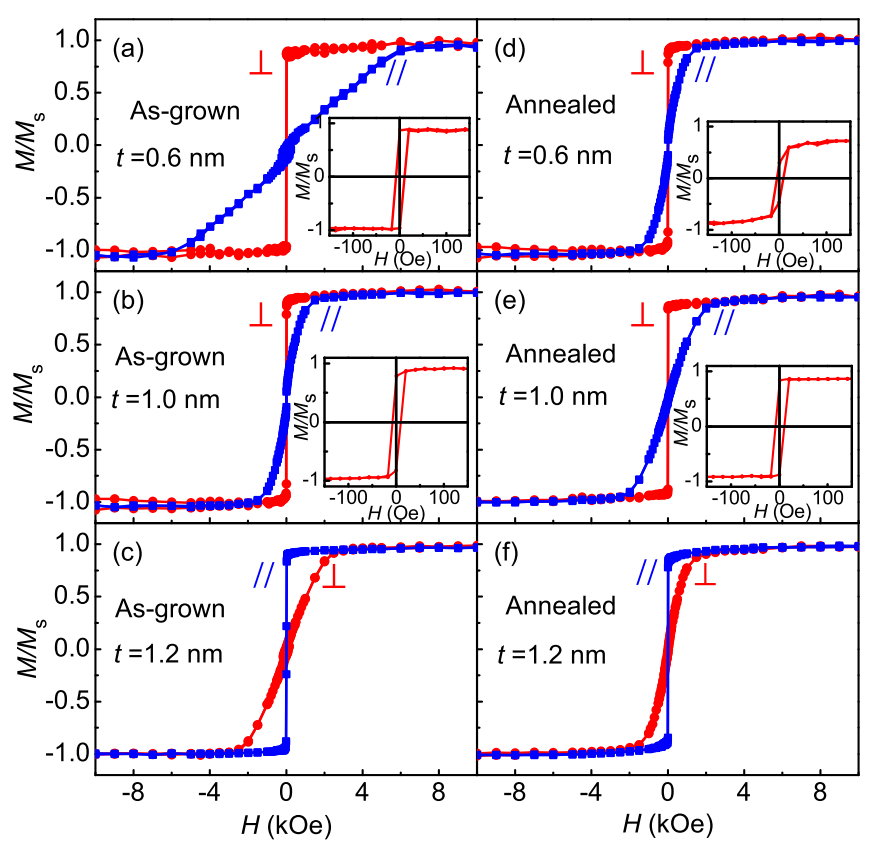

FIG. 1. Perpendicular (red line with solid circles) and in-plane (blue line with solid squares) $M / M_{\mathrm{s}}-H$ loops of $\mathrm{Ta}(5) / \mathrm{CoFeB}(t) / \mathrm{MgAl}_{2} \mathrm{O}_{4}(2) / \mathrm{Ta}(5)$ (in nm) multilayer before and after annealing at $300^{\circ} \mathrm{C}$ with $t=0.6 \mathrm{~nm}$ (a) and (d), $1.0 \mathrm{~nm}$ (b) and (e), $1.2 \mathrm{~nm}$ (c) and (f), respectively. The inset figures show the enlarged perpendicular loops.

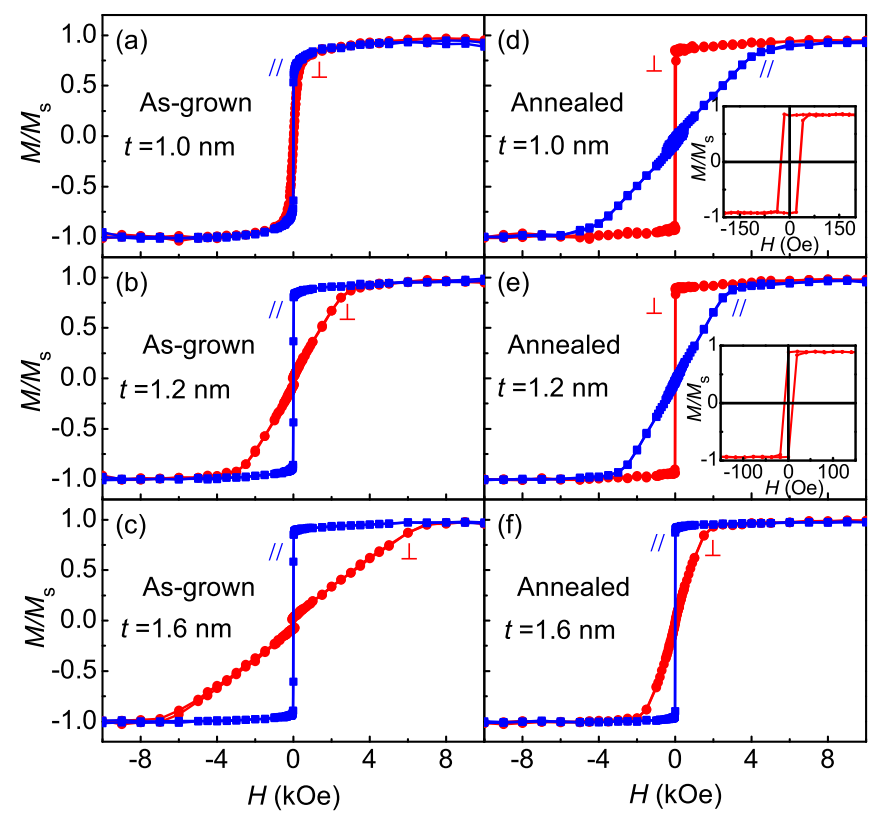

FIG. 2. Perpendicular (red line with solid circles) and in-plane (blue line with solid squares) $M / M s-H$ loops of $\mathrm{Ta}(5) / \mathrm{MgAl}_{2} \mathrm{O}_{4}(2) / \mathrm{CoFeB}(t) / \mathrm{Ta}(5)$ (in nm) multilayer before and after annealed at $300^{\circ} \mathrm{C}$ with $t=1.0 \mathrm{~nm}$ (a) and (d), $1.2 \mathrm{~nm}$ (b) and (e), $1.6 \mathrm{~nm}$ (c) and (f), respectively. The inset figures show the enlarged perpendicular loops.

component with decreasing $t$. After annealing at $300^{\circ} \mathrm{C}$, the PMA is enhanced significantly, see Figs. 2(d)-2(f). For the samples with $t=1.0$ and $1.4 \mathrm{~nm}$, the easy axis turns from inplane to out-of- plane showing squared-well hysteresis loops with $\mathrm{SQ}=1$. While for sample with $t=1.6 \mathrm{~nm}$, the easy axis is still in plane, but the perpendicular saturation field decreases from 7000 to 2000 Oe after annealing, indicating an enhanced contribution from PMA. These results suggest that annealing process can improve PMA significantly and is essential for top electrodes to achieve good PMA.

Figs. 3(a) and 3(b) show the saturation magnetic moment per unit area as a function of nominal thickness of $\mathrm{CoFeB}$ for bottom and top electrodes, respectively. The magnetic dead layer (MDL) and saturation magnetization of $\mathrm{CoFeB}$ can be extracted by linearly fitting the data. The intercept of $x$-axis and slope of the fitted lines are used to determine the thickness $t_{\mathrm{d}}$ of MDL and saturation magnetization $\left(M_{\mathrm{s}}\right)$ of $\mathrm{CoFeB}$. Generally, three kinds of magnetic anisotropy including bulk anisotropy, shape anisotropy, and interfacial anisotropy contribute to the effective anisotropy in multilayer structure. ${ }^{25}$ Moreover, annealing treatment can impact the magnetic anisotropy of the structure by affecting crystallization of ferromagnetic layer, interface roughness, inter-diffusion, and stress. ${ }^{25}$ In the ultra thin $\mathrm{CoFeB}$ film stacks, the interfacial anisotropy plays an important role. Thus, the effective anisotropy $\left(K_{\mathrm{u}}\right)$ can be described as

$$
K_{\mathrm{u}}=K_{\mathrm{b}}-2 \pi M_{\mathrm{s}}^{2}+\frac{K_{\mathrm{i}}}{t_{\mathrm{eff}}},
$$

where $K_{\mathrm{u}}$ can be estimated by $K_{\mathrm{u}}=H_{\mathrm{s}} M_{\mathrm{s}} / 2$ with $H_{\mathrm{s}}$ for saturation field along hard axis and $M_{\mathrm{s}}$ for saturation magnetization; $K_{\mathrm{b}}$ and $K_{\mathrm{i}}$ represent bulk and interfacial anisotropy; $-2 \pi M_{\mathrm{s}}{ }^{2}$ is the shape anisotropy, and $t_{\mathrm{eff}}$ indicates effective thickness of $\mathrm{CoFeB}$, defined as $t_{\mathrm{eff}}=t-t_{\mathrm{d}}$ with $t_{\mathrm{d}}$ for 

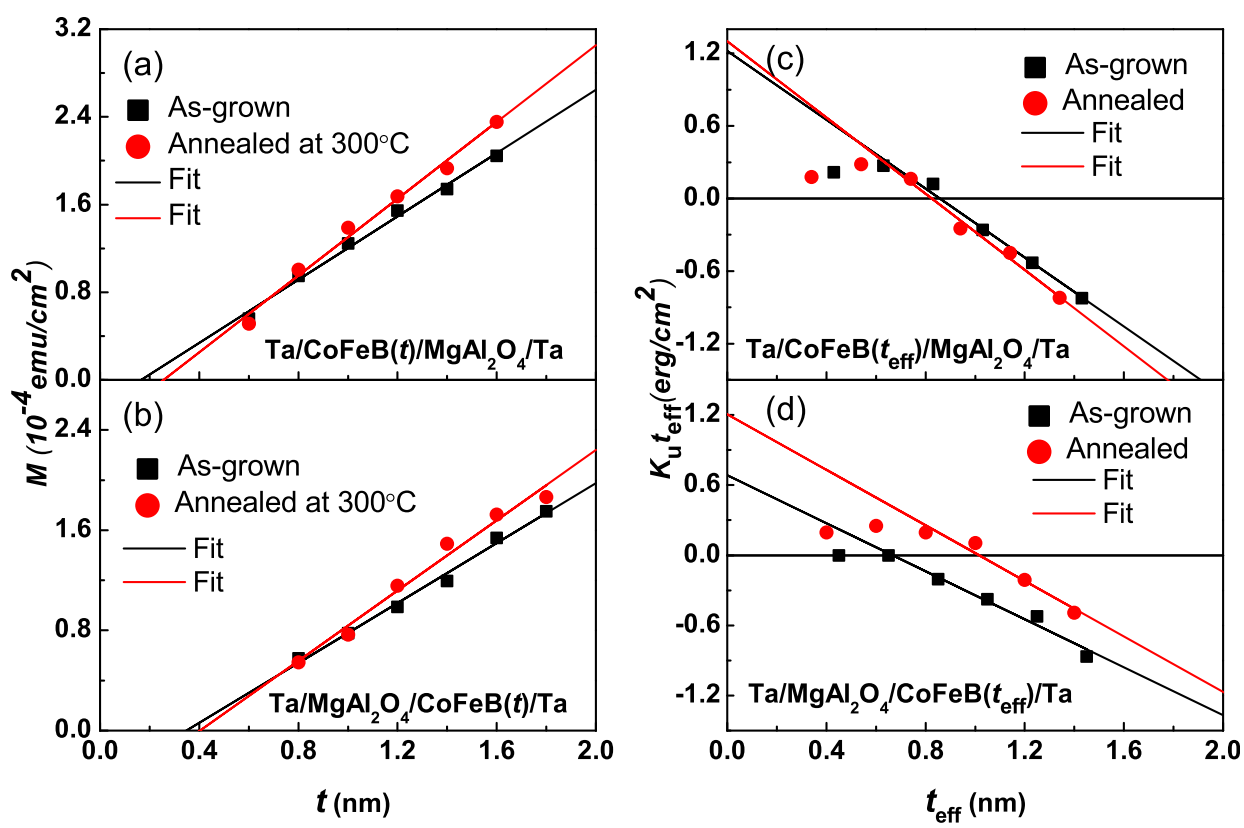

FIG. 3. (a) and (b) Magnetic moment per unit area dependence on nominal thickness $t$ for $\mathrm{Ta}(5) / \mathrm{CoFeB}(t) / \mathrm{MgAl}_{2} \mathrm{O}_{4}$ (2)/Ta (5) and $\mathrm{Ta}(5) / \mathrm{MgAl}_{2} \mathrm{O}_{4}$ (2)/ $\mathrm{CoFeB}(t) / \mathrm{Ta}(5)$ (in $\mathrm{nm}$ ), respectively. (c) and (d) The product of perpendicular anisotropy $K_{\mathrm{u}}$ and effective thickness $t_{\mathrm{eff}}$ as a function of $t_{\mathrm{eff}}$ for Ta (5)/ $\mathrm{CoFeB}\left(t_{\text {eff }}\right) / \mathrm{MgAl}_{2} \mathrm{O}_{4}$ (2)/Ta (5) and $\mathrm{Ta}(5) / \mathrm{MgAl}_{2} \mathrm{O}_{4}(2) / \mathrm{CoFeB}\left(t_{\text {eff }}\right) / \mathrm{Ta}$ (5) (in $\mathrm{nm}$ ), respectively. The effective thickness is defined as $t_{\text {eff }}=t-t_{\mathrm{d}}$, where $t_{\mathrm{d}}$ is dead layer thickness for $\mathrm{CoFeB}$. Solid squares and circles represent experimental data of as deposited and annealed states, respectively. Linear fit to the data are shown by the solid lines. thickness of MDL for CoFeB. The results for bottom and top electrodes are illustrated in Figs. 3(c) and 3(d). To estimate the interfacial contributions to the effective anisotropy, the curves of $K_{\mathrm{u}} t_{\mathrm{eff}}$ as a function of $t_{\mathrm{eff}}$ are linearly fitted. All the fitting results are summarized in Table I.

$\mathrm{CoFeB}$ thin films deposited on bottom and top of $\mathrm{MgAl}_{2} \mathrm{O}_{4}$ layer show different behavior and have different annealing effects. The saturation magnetization of $\mathrm{CoFeB}$ for bottom electrode is larger than that of top electrode and it increases after annealing due to crystallization of $\mathrm{CoFeB}$. The MDL is observed in both bottom and top electrodes. The thickness of MDL increases from 0.17 to $0.26 \mathrm{~nm}$ for bottom electrode and 0.35 to $0.40 \mathrm{~nm}$ for top electrode after annealing process, which can be attributed to inter-diffusion between $\mathrm{Ta}$ and $\mathrm{CoFeB}$ at the interface during annealing. ${ }^{23,24}$ The interfacial anisotropy $K_{\mathrm{i}}$, which originates from hybridization of transition metal $3 d$ and O $2 p$ orbitals ${ }^{20,21}$ changes slightly after annealing for bottom electrode, while it is almost doubled for top electrode. It should be pointed out that though the $\mathrm{Ta} / \mathrm{CoFeB}$ interface makes contribution to the PMA, ${ }^{6,8}$ the intermixing of $\mathrm{Ta}$ and $\mathrm{CoFeB}$ is not beneficial to the PMA. ${ }^{6,9}$ Since the annealing process further promotes this intermixing, the enhancement of $K_{\mathrm{i}}$ should be attributed to the interface between $\mathrm{CoFeB}$ and $\mathrm{MgAl}_{2} \mathrm{O}_{4}$. This means that strong Fe-O or Co-O bonding exists in bottom electrodes even in the as-grown state and the annealing process only result in a slight increase in $K_{\mathrm{i}}$. While for top

TABLE I. Results extracted from linear fitting of experimental data for bottom and top electrode in the as-grown and annealed states.

\begin{tabular}{lcccccc}
\hline \hline & \multicolumn{2}{c}{ Bottom electrode } & & \multicolumn{2}{c}{ Top electrode } \\
\cline { 2 - 3 } \cline { 6 - 6 } & As-grown & Annealed & & As-grown & Annealed \\
\hline$M_{\mathrm{s}}\left(\mathrm{emu} / \mathrm{cm}^{3}\right)$ & 1443 & 1751 & & 1195 & 1402 \\
$t_{\mathrm{d}}(\mathrm{nm})$ & 0.17 & 0.26 & & 0.35 & 0.4 \\
$K_{\mathrm{i}}\left(\mathrm{erg} / \mathrm{cm}^{2}\right)$ & 1.22 & 1.30 & & 0.68 & 1.21 \\
$2 \pi M_{\mathrm{s}}{ }^{2}\left(10^{6} \mathrm{erg} / \mathrm{cm}^{3}\right)$ & 13.1 & 19.3 & & 8.97 & 12.3 \\
$K_{\mathrm{b}}\left(10^{6} \mathrm{erg} / \mathrm{cm}^{3}\right)$ & -1.10 & 3.60 & & -1.33 & 0.4 \\
\hline \hline
\end{tabular}

electrode, the weak Fe-O or Co-O bonding is responsible for the small value of $K_{\mathrm{i}}$ in as-grown state, and annealing process significantly enhances $K_{\mathrm{i}}$ due to the appearance of significant density of $\mathrm{Fe}-\mathrm{O}$ and $\mathrm{Co}-\mathrm{O}$ bonding. ${ }^{22}$ The interfacial anisotropy is comparable with that of $\mathrm{Ta} / \mathrm{CoFeB} / \mathrm{MgO}$ structures, ${ }^{5}$ and the anisotropy energy is high enough to ensure high thermal stability. Note that $K_{\mathrm{b}}$ for both top and bottom electrodes changes sign after annealing, which is beneficial to PMA. This probably originates from magneto-elastic anisotropy, which is also considered as a factor for PMA. ${ }^{25,26}$

After PMA was obtained in both bottom and top electrodes, p-MTJs were fabricated and studied. The RBS was used to characterize the element distribution and stoichiometry of the barrier in MTJ. Figs. 4(a) and 4(b) show RBS for p-MTJs' core structure of $\mathrm{CoFeB}(1.0) / \mathrm{MgAl}_{2} \mathrm{O}_{4}(2) / \mathrm{CoFeB}(1.2)$ (numbers are nominal thickness in nanometer) at as-grown state and annealed at $300^{\circ} \mathrm{C}$, respectively. The barrier at the bottom interface is stoichiometric $\mathrm{MgAl}_{2} \mathrm{O}_{4}$, while there is $\mathrm{O}$ vacancy at the top interface. The asymmetrical distribution of $\mathrm{O}$ on bottom and top interface accounts for the different magnetic behaviors of bottom and top electrodes. For the asgrown state, there exist $\mathrm{Ta}$ and $\mathrm{CoFeB}$ intermixing on both top and bottom interfaces, causing magnetic dead layer of $\mathrm{CoFeB}$. Compared with bottom $\mathrm{CoFeB}$ layer, the content of $\mathrm{Co}$ and $\mathrm{Fe}$ is less in top $\mathrm{CoFeB}$, resulting in a smaller $M_{s}$ of top CoFeB. Moreover, top Ta diffuses into the barrier, which is not beneficial to the TMR. For the sample annealing at $300{ }^{\circ} \mathrm{C}$, the intermixing of $\mathrm{Ta}$ and $\mathrm{CoFeB}$ increases on the bottom interface, which accounts for the increased thickness of MDL. Figs. 4(c) and 4(d) show the normalized perpendicular and in-plane hysteresis curves for as-grown and annealed MTJs, respectively. For the as-grown MTJ, $M /$ $M_{\mathrm{s}}-H$ loops show sharp switching near zero field for both $H$ in-plane and out-of-plane, indicating that only the bottom electrode exhibit PMA. After annealing at $300^{\circ} \mathrm{C}$, a full perpendicular MTJ was obtained. Moreover, according to the inset figures in Figs. 4(c) and 4(d), the switching field of the bottom electrode is about $10 \mathrm{Oe}$, which is smaller than that of the top electrode, which is about $40 \mathrm{Oe}$, resulting in an 

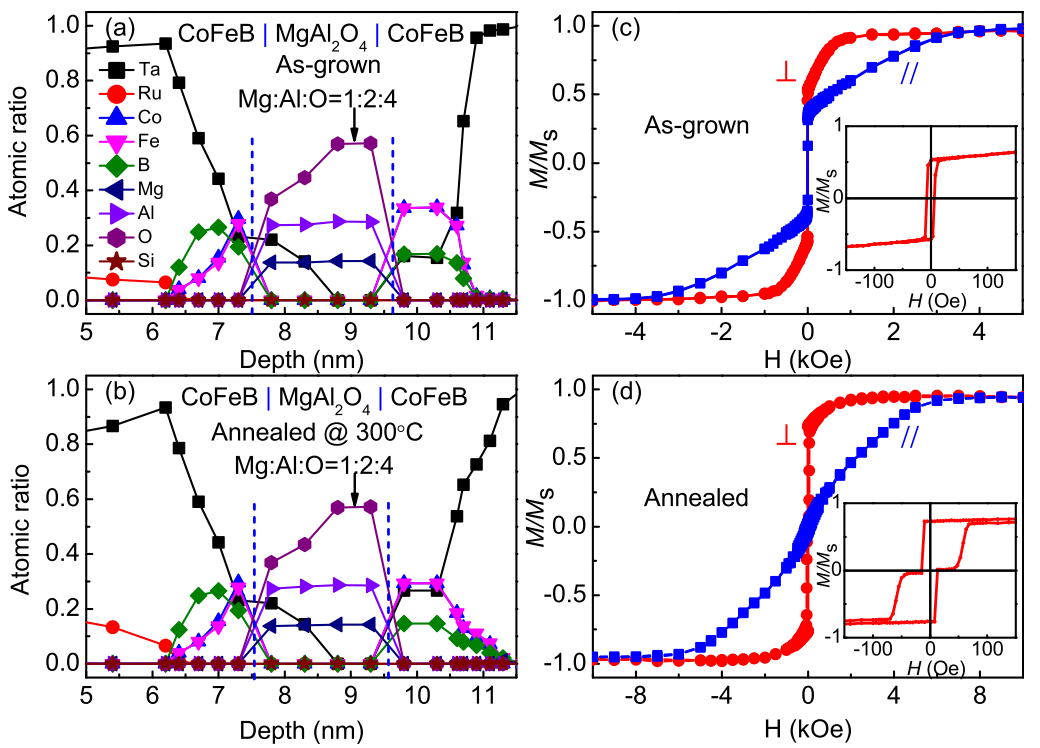

FIG. 4. RBS of MTJs's core structure of $\mathrm{CoFeB} /$ $\mathrm{MgAl}_{2} \mathrm{O}_{4} / \mathrm{CoFeB}$ trilayers in the (a) as-grown and (b) annealed states. Normalized magnetic hysteresis loops for the as-grown (c) and annealed (d) MTJs, where the red line with solid circles and blue line with solid squares represent $H$ out of plane and in plane, respectively. The inset figures in (c) and (d) show the enlarged perpendicular loops. antiparallel step in $M-H$ curve, which is necessary for MTJs to achieve the parallel and antiparallel magnetic configurations.

Figs. 5(a)-5(c) display TMR $-H$ curves at $T=300 \mathrm{~K}$, $150 \mathrm{~K}$, and $10 \mathrm{~K}$, respectively. Note that the coercivity of bottom and top electrodes are increased after patterned into micro junctions and enhanced significantly at $T=10 \mathrm{~K}$. The TMR ratio of about $36 \%$ at room temperature and about $63 \%$ at low temperature are obtained. For $T=150 \mathrm{~K}$, the TMR ratio decreased to $27 \%$ and there exist only one switch without a complete antiparallel platform in positive field. The resistance in parallel $\left(R_{\mathrm{P}}\right)$ and antiparallel $\left(R_{\mathrm{AP}}\right)$ state and TMR ratio as a function of temperature $T$ are shown in Fig. 5(d). The resistance-area product (RA) of the p-MTJ in parallel state at room temperature is $1.4 \times 10^{5} \Omega \mu \mathrm{m}^{2}$. With decreasing temperature, $R_{\mathrm{P}}$ increases monotonously while $R_{\mathrm{AP}}$ shows a dip around $150 \mathrm{~K}$, resulting a dip in TMR. The antiferromagnet of ferrimagnet may form at the interface of ferromagnetic layer and tunnel barrier due to the inter-diffusion of $\mathrm{Fe}, \mathrm{Co}$,
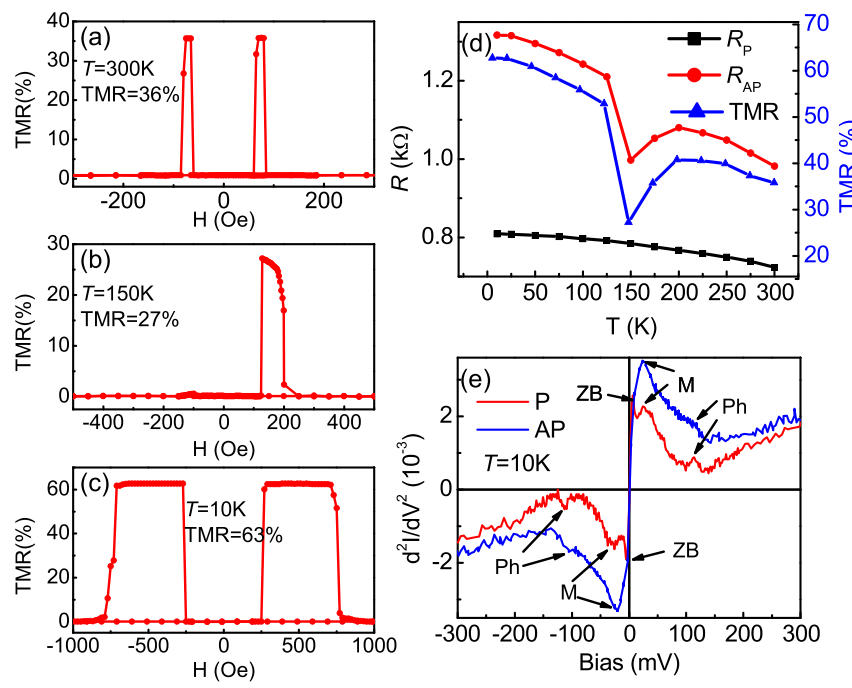

FIG. 5. TMR- $H$ curves of MTJ at $T=300 \mathrm{~K}$ (a), $150 \mathrm{~K}$ (b), and $10 \mathrm{~K}$ (c), respectively. Temperature dependence of resistance $R_{\mathrm{P}}, R_{\mathrm{AP}}$, and TMR ratio and IETS at T $=10 \mathrm{~K}$ were shown in (d) and (e), respectively. and $\mathrm{O}$, and induce unidirectional anisotropy by intrinsic exchange bias, resulting in asymmetric behavior at $T=150 \mathrm{~K}$. However, there exists no unidirectional anisotropy in the MTJ structures, which has been confirmed by the magnetic hysteresis loops of top and bottom $\mathrm{CoFeB}$ layers at different temperature (not shown here). Thus, the asymmetric behavior at $\mathrm{T}=150 \mathrm{~K}$ is not caused by unidirectional anisotropy. Note that there is no well-defined antiparallel state and the magnetization switch gradually in positive field. The asymmetric behavior may be attributed to the small coercivity difference of top and bottom electrodes because of the different temperature dependence of coercivity ${ }^{28}$ and coupling of two magnetic layers, which result in simultaneous switching and unstable antiparallel state. The phenomenon has also been observed previously. ${ }^{27}$ In order to characterize intrinsic excitations in the p-MTJ, inelastic electrontunneling spectroscopy (IETS) has been measured, as shown in Fig. 5(e). Three peaks, including zero-bias anomaly (ZB) due to magnetic impurity scattering, magnon excitation (M) at the interface of ferromagnetic electrode and barrier, and phonon excitation $(\mathrm{Ph})$ in barrier, are usually found in $\mathrm{AlO}_{\mathrm{x}}$ and $\mathrm{MgO}$ based MTJs. ${ }^{29}$ In this work, ZB locating at $5 \mathrm{mV}$ and $\mathrm{M}$ locating at $25 \mathrm{mV}$ are identified, which is comparable with in-plane MTJs. ${ }^{29}$ The peak at voltage of about $115 \mathrm{mV}$ is identified as $\mathrm{Ph}$, originating from phonon excitation in $\mathrm{MgAl}_{2} \mathrm{O}_{4}$ barrier.

Compared with $\mathrm{MgO}$ based p-MTJs, the obtained TMR ratio in our work is lower, which can be attributed to the poor quality of the barrier. As can be seen from the RBS results, the barrier is not perfect stoichiometric $\mathrm{MgAl}_{2} \mathrm{O}_{4}$, thus, the barrier is not perfect spinel oxide with spin-filter properties as the same of $\mathrm{MgO}$, resulting in an incoherent tunneling process. In addition, the element of $\mathrm{Ta}$ is detected at top interface, indicating an ambiguous interface, which also results in low TMR. Further improvement of the quality of the barrier is needed to enhance the TMR ratio of $\mathrm{MgAl}_{2} \mathrm{O}_{4}$ based p-MTJ.

In summary, the magnetic characteristics of $\mathrm{CoFeB}$ thin films in $\mathrm{Ta} / \mathrm{CoFeB} / \mathrm{MgAl}_{2} \mathrm{O}_{4} / \mathrm{Ta}$ and $\mathrm{Ta} / \mathrm{MgAl}_{2} \mathrm{O}_{4} / \mathrm{CoFeB} / \mathrm{Ta}$ structures have been systematically investigated. Bottom electrodes show PMA in the as-grown state and change 
slightly after annealing at $300^{\circ} \mathrm{C}$, while for top electrodes the annealing process is essential to achieve PMA. The upper limits for CoFeB thickness possessing PMA for bottom and top electrodes are $1.0 \mathrm{~nm}$ and $1.4 \mathrm{~nm}$, respectively. The MDL exists in both electrodes and increases after annealing. The different magnetic behaviors of $\mathrm{CoFeB}$ in bottom and top electrodes can be attributed to asymmetrical distribution of $\mathrm{O}$ in bottom and top interfaces. Perpendicular $\mathrm{CoFeB} /$ $\mathrm{MgAl}_{2} \mathrm{O}_{4} / \mathrm{CoFeB}$ MTJs have been fabricated and antiparallel platform can be obtained after annealing at $300^{\circ} \mathrm{C}$. TMR ratio of $36 \%$ at $300 \mathrm{~K}$ and $63 \%$ at $10 \mathrm{~K}$ are observed. Three kinds of intrinsic excitations, including $\mathrm{ZB}, \mathrm{M}$, and $\mathrm{Ph}$, have been identified by IETS in p-MTJs.

The work was supported by the State Key Project of Fundamental Research of Ministry of Science and Technology [MOST, Nos. 2010CB934401 and 2014AA032904], and the National Natural Science Foundation [NSFC, Grant Nos. 51229101, 11174341, and 11222432].

${ }^{1}$ J.-G. Zhu and C. Park, Mater. Today 9, 36 (2006).

${ }^{2}$ R. Sbiaa, H. Meng, and S. N. Piramanayagam, Phys. Status Solidi RRL 5, 413 (2011).

${ }^{3}$ A. Brataas, A. D. Kent, and H. Ohno, Nat. Mater. 11, 372 (2012).

${ }^{4}$ A. V. Khvalkovskiy, D. Apalkov, S. Watts, R. Chepulskii, R. S. Beach, A. Ong, X. Tang, A. Driskill-Smith, W. H. Butler, P. B. Visscher, D. Lottis, E. Chen, V. Nikitin, and M. Krounbi, J. Phys. D: Appl. Phys. 46, 074001 (2013).

${ }^{5}$ S. Ikeda, K. Miura, H. Yamamoto, K. Mizunuma, H. D. Gan, M. Endo, S. Kanai, J. Hayakawa, F. Matsukura, and H. Ohno, Nat. Mater. 9, 721 (2010).

${ }^{6}$ D. C. Worledge, G. Hu, D. W. Abraham, J. Z. Sun, P. L. Trouilloud, J. Nowak, S. Brown, M. C. Gaidis, E. J. O'Sullivan, and R. P. Robertazzi, Appl. Phys. Lett. 98, 022501 (2011).

${ }^{7}$ W. X. Wang, Y. Yang, H. Naganuma, Y. Ando, R. C. Yu, and X. F. Han, Appl. Phys. Lett. 99, 012502 (2011).

${ }^{8}$ T. Liu, J. W. Cai, and L. Sun, AIP Adv. 2, 032151 (2012).

${ }^{9}$ J. Sinha, M. Hayashi, A. J. Kellock, S. Fukami, M. Yamanouchi, H. Sato, S. Ikeda, S. Mitani, S. Yang, S. S. P. Parkin, and H. Ohno, Appl. Phys. Lett. 102, 242405 (2013).
${ }^{10}$ J. G. Alzate, P. K. Amiri, G. Yu, P. Upadhyaya, J. A. Katine, J. Langer, B. Ocker, I. N. Krivorotov, and K. L. Wang, Appl. Phys. Lett. 104, 112410 (2014).

${ }^{11}$ S. S. P. Parkin, C. Kaiser, A. Panchula, P. M. Rice, B. Hughes, M. Samant, and S.-H. Yang, Nat. Mater. 3, 862 (2004).

${ }^{12}$ S. Yuasa, T. Nagahama, A. Fukushima, Y. Suzuki, and K. Ando, Nat. Mater. 3, 868 (2004).

${ }^{13}$ S. Ikeda, J. Hayakawa, Y. Ashizawa, Y. M. Lee, K. Miura, H. Hasegawa, M. Tsunoda, F. Matsukura, and H. Ohno, Appl. Phys. Lett. 93, 082508 (2008).

${ }^{14}$ H. Sukegawa, H. X. Xiu, T. Ohkubo, T. Furubayashi, T. Niizeki, W. H. Wang, S. Kasai, S. Mitani, K. Inomata, and K. Hono, Appl. Phys. Lett. 96, 212505 (2010).

${ }^{15}$ J. Zhang, X.-G. Zhang, and X. F. Han, Appl. Phys. Lett. 100, 222401 (2012).

${ }^{16}$ H. F. Liu, Q. L. Ma, S. Rizwan, D. P. Liu, S. G. Wang, and X. F. Han, IEEE Trans. Mag. 47, 2716 (2011).

${ }^{17}$ Y. Miura, S. Muramoto, K. Abe, and M. Shirai, Phys. Rev. B 86, 024426 (2012).

${ }^{18}$ H. Sukegawa, Y. Miura, S. Muramoto, S. Mitani, T. Niizeki, T. Ohkubo, K. Abe, M. Shirai, K. Inomata, and K. Hono, Phys. Rev. B 86, 184401 (2012).

${ }^{19}$ H. Sukegawa, S. Mitani, T. Ohkubo, K. Inomata, and K. Hono, Appl. Phys. Lett. 103, 142409 (2013).

${ }^{20}$ R. Shimabukuro, K. Nakamura, T. Akiyama, and T. Ito, Physica E 42, 1014 (2010).

${ }^{21}$ H. Yang, M. Chshiev, B. Dieny, J. Lee, A. Manchon, and K. Shin, Phys. Rev. B 84, 054401 (2011).

${ }^{22}$ A. Manchon, C. Ducruet, L. Lombard, S. Auffret, B. Rodmacq, B. Dieny, S. Pizzini, J. Vogel, V. Uhlí, M. Hochstrasser, and G. Panaccione, J. Appl. Phys. 104, 043914 (2008).

${ }^{23}$ M. Kowalewski, W. H. Butler, N. Moghadam, G. M. Stocks, T. C. Schulthess, K. J. Song, J. R. Thompson, A. S. Arrott, T. Zhu, J. Drewes, R. R. Katti, M. T. McClure, and O. Escorcia, J. Appl. Phys. 87, 5732 (2000).

${ }^{24}$ S. Y. Jang, C. Y. You, S. H. Lim, and S. R. Lee, J. Appl. Phys. 109, 013901 (2011).

${ }^{25}$ M. T. Johnson, P. J. H. Bloemen, F. J. A. den Broeder, and J. J. de Vries, Rep. Prog. Phys. 59, 1409 (1996).

${ }^{26}$ H. Meng, W. H. Lum, R. Sbiaa, S. Y. H. Lua, and H. K. Tan, J. Appl. Phys. 110, 033904 (2011).

${ }^{27}$ G. Feng, H. C. Wu, J. F. Feng, and J. M. D. Coey, Appl. Phys. Lett. 99, 042502 (2011).

${ }^{28}$ M. C. Tsai, C. W. Cheng, C. C. Tsai, and G. Chern, J. Appl. Phys. 113, 17C118 (2013).

${ }^{29}$ V. Drewello, M. Schäfers, O. Schebaum, A. Khan, J. Münchenberger, J. Schmalhorst, G. Reiss, and A. Thomas, Phys. Rev. B 79, 174417 (2009). 